\title{
Software Framework to Quantify Pulmonary Vein Isolation Atrium Scar Tissue
}

\author{
José A Solís-Lemus ${ }^{1}$, Orod Razeghi ${ }^{1}$, Caroline Roney ${ }^{1}$, Iain Sim $^{1}$, Rahul Mukherjee ${ }^{1}$, Steven \\ Williams ${ }^{1}$, Mark O’Neill², Steven Niederer ${ }^{1}$ \\ ${ }^{1}$ School of Biomedical Engineering and Imaging Sciences, King's College London, UK \\ 'Guy's and St Thomas' Hospital NHS Foundation Trust, Cardiology Department, London, UK
}

\begin{abstract}
Pulmonary vein isolation (PVI) is a recommended treatment for drug refractory atrial fibrillation $(A F)$. The ablation causes non-conductive scar tissue that separates the body of the atrium from regions around the pulmonary veins that trigger AF. This scar tissue can be seen in the atrium using cardiac MR. We aim to develop a systematic workflow to quantify the location and size of scar tissue due to PVI from CMR images to evaluate ablation procedures. Input meshes were created from segmented late-gadolineum-enhanced (LGE) cardiac magnetic resonance (CMR) scans with scalar values at each node representing the signal intensity of the scar. The software has three features: (i) Calculating the area of the scar tissue, (ii) assessing ablation lesions to identify the percentage of the pulmonary vein encircled by scar and the number of gaps in it, and (iii) comparing pre- and postablation scar tissues qualitatively. Six patients were assessed as a proof of concept, where patients had undergone pre- and post-ablation scans. In all tests, an increase in fibrotic tissue was found, from averages of $2+1.9 \%$ and $36+18 \%$. Post-ablation lesions were assessed showing an average of $75+12 \%$ pulmonary vein encirclement, with gaps ranging from 2 to 4 . The software presented is a semi-automated, user friendly framework where users are able to assess an ablation procedure.
\end{abstract}

\section{Introduction}

Pulmonary vein isolation (PVI) is a common ablation procedure to isolate abnormal electrical signals occurring in the pulmonary veins. A successful ablation produces a lesion encircling the veins and stopping activation propagating from the pulmonary veins to the body of the atrium[1]. Late-gadolineum-enhanced (LGE) cardiac magnetic resonance (CMR) scans are the standard tool for imaging of chronic scar [2].

There are few available methods to robustly assess CMR ablation lesions, in many cases, visual inspection is required [3]-[5]. In [1], a fully automated approach to quantify the ablation is presented. However, the high variability in pulmonary veins' (PV) morphology limits this approach to common patients with 4 PV configurations, as the method requires a very specific parcellation of the atrium.

This work presents a set of software tools to quantify features in the scar tissue within the left atrium, with emphasis on comparing the status of the scar before and after an ablation procedure for PVI. The aim is to produce a set of semi-automatic methods which, with minimal user input, can effect reliable and reproducible measurements. The software, called Advanced Scar Calculations is built on top of the open source software CemrgApp [6].

\section{Materials \& Methods}

The Cardio Electro-Mechanics Research Group Application (CemrgApp) [6] is an open source software platform providing an integrated custom environment for developing computer vision tools.

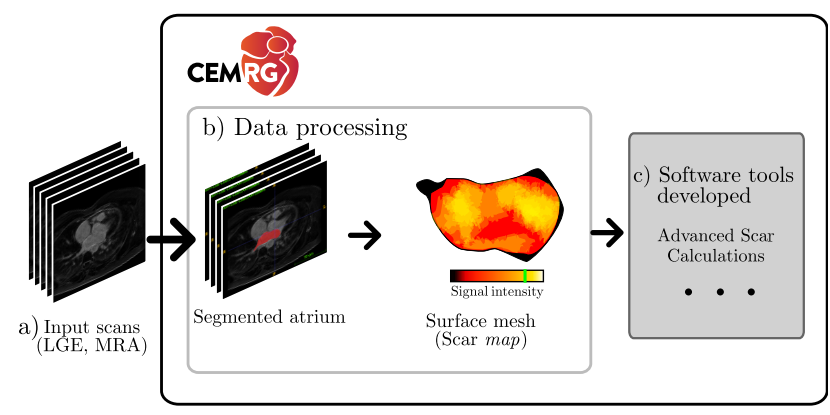

Figure 1. Developing of Advanced Scar Calculations tools on top of CemrgApp's atrial scar quantification toolkit. Input scans (a) are loaded into the application and processed (b) to produce a surface mesh with point values corresponding to the intensity of the scar signal calculated from the LGE-CMR scans. The developed software (c) assesses and compares instances of preand post-ablation scans.

CemrgApp was used as a platform to develop the tools and to process the input data. The LGE-CMR scan is loaded into the application, where it is segmented. A surface mesh is generated from the segmentation, where the scalar values of each node corresponds to the signal intensity for the scar, as documented in [7]. 

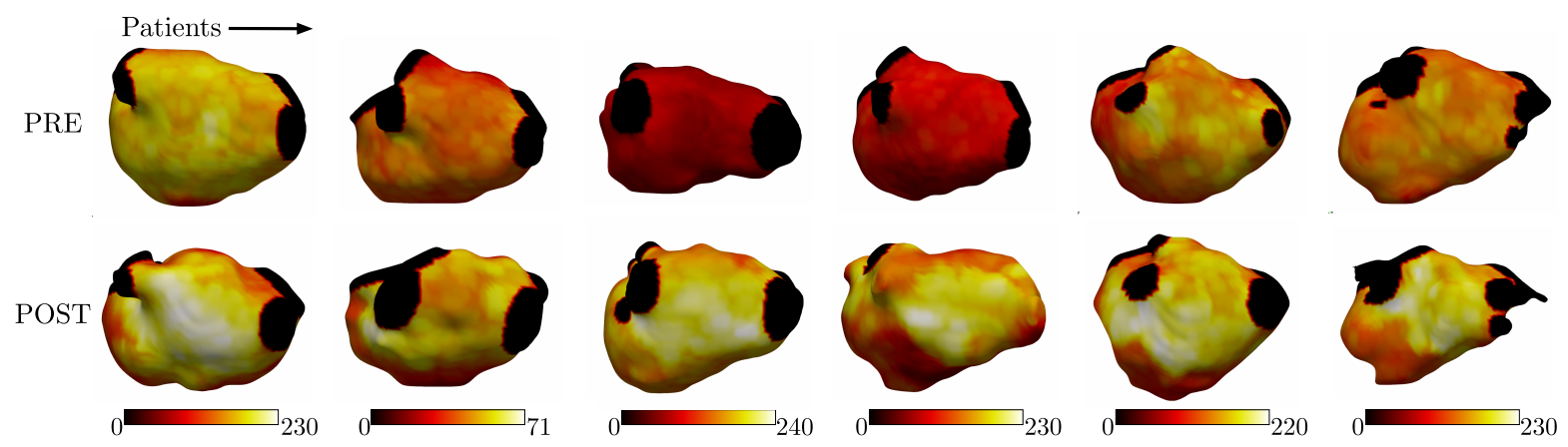

Figure 2. Generation of pre- and post-ablation scar maps for Advanced Scar Calculations. Top row: Pre-ablation scar maps. Bottom row: Post-ablation scar maps. The signal intensities and colour maps shown are calculated per patient. Notice the differences in shapes and sizes, even in the same patient

The software tools described in this paper are called Advanced Scar Calculations, as they complement CemrgApp's atrial scar tissue functionalities. Figure 1 presents the flow of information from the acquired volumes, through the processing into surface shells, and into the Advanced Scar Calculations block where three functionalities were developed: (i) Scar area, relative to a chosen threshold, (ii) assessing ablation lesions and (iii) comparison of pre- and post- ablation scar tissues.

\subsection{Input data and scar map generation}

Scans from six patients were assessed, as a proof of concept, where patients had undergone pre- and postablation scans. The procedure for acquiring each patient's scans is thoroughly documented in [5], and [6]. The volumes generated are loaded into CemrgApp and resampled to be isometric [6].

To generate the scar map, the Scar Quantification pipeline of CemrgApp was used as described in previous studies [7], [8]. The pipeline consists of a semi-automatic segmentation of the MRA scan. The segmentation is then registered to the LGE-CMR scan. A surface mesh is generated from the aligned segmentation. Next, the pulmonary veins, left atrial appendage, and mitral valve were clipped from the mesh. Finally, the signal intensities on the LGE image were projected onto the mesh, using a maximum intensity projection on the direction of the normal of the mesh, the signal taken within $3 \mathrm{~mm}$ of the endocardial border.

Figure 2 shows the scar maps used as input to the Advanced Scar Calculations software. Since the scar signal intensity values do not fall under a specific range, the scar signal is assessed via a threshold relative to the mean intensity of the blood pool. Common methods to calculate the thresholds include a percentage of the image intensity ratio (IIR) and 3.3 standard deviations (SD) above the mean (M) blood pool signal (M + 3.3SD) [7] [10], the latter was chosen for all the cases in this work.

\subsection{Advanced Scar Calculations}

The software consists of a graphical interface, where a scar map is presented to the user in an interactive rendered window, and user input elements. The three main methods are executed upon pressing the corresponding button. Some secondary functionalities were incorporated to facilitate user interaction such as a shell selector between pre- and post- ablation and a threshold selector. Figure 3 shows a graphical representation of the proposed tools.

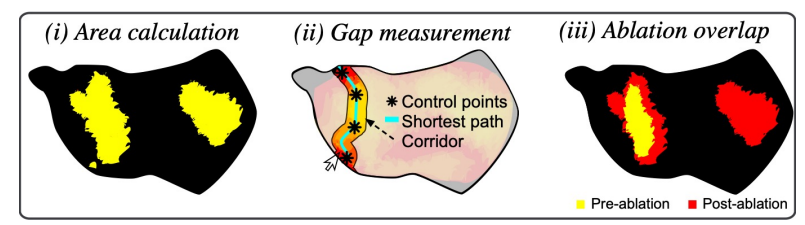

Figure 3. Advanced Scar Calculations diagram.

i) Calculating the area of the scar tissue. The area of the scar tissue is calculated by adding the areas of the triangles in the mesh where the signal value is higher than the threshold. The user is able to try different thresholds relevant in the literature and calculate the area for those.

ii) Assessing ablation lesions. Following a procedure similar to the one shown in [8], an encircling corridor around the veins is generated via user-defined points. The points are joined by the Dijkstra algorithm, calculating the shortest path weighted by the signal intensity values. The user also selects the width of the corridor. Gaps in the corridor are defined as sections of the corridor where the scar signal intensities are below the chosen threshold.

iii) Comparing pre- and post-ablation scar tissues. A visualization of both scar maps is available through a superposition of the two meshes and corresponding segmented scar tissues. As observed in Figure 2, the shapes of the meshes do not match from the pre- and post-ablation scans. To overcome such a problem, the two scans were aligned at their centres of mass, then the pre-ablation values were projected onto the post-ablation shell. 


\section{Results}

The three methods were applied to the six scar maps previously described. In results presented with all the patients, the order patients presented (01 to 06) corresponds to the order of scar maps presented in Figure 2. In all cases, a threshold value of 3.3SD above the mean (M) was chosen for the subsequent methods. The results for the scar scores in pre and post-ablation, as well as the ratio of pre-ablation to post-ablation scar are presented in Table 1 .

Table 1. Percentage of scar comparison. Patients with a postablation percentage higher than $30 \%$ are highlighted in bold. The bottom row shows the mean (M) and standard deviation (SD) of the measurements.

\begin{tabular}{llll}
\hline \multicolumn{4}{c}{ Scar tissue \% } \\
\hline Patient & Pre-ablation & Post-ablation & Pre/Post \% \\
\hline 01 & 0.06 & $\mathbf{3 5 . 9}$ & 0.17 \\
02 & 5.52 & $\mathbf{3 4 . 3 2}$ & 16.08 \\
03 & 1.22 & $\mathbf{6 9 . 4 9}$ & 1.76 \\
04 & 1.54 & 13.26 & 11.61 \\
05 & 2.83 & $\mathbf{3 6 . 2 2}$ & 7.81 \\
06 & 0.93 & 27.89 & 3.33 \\
\hline $\mathrm{M}+\mathrm{SD}$ & $2+1.9$ & $36.2+18.5$ & \\
\hline
\end{tabular}

The encirclement corridor was generated in the postablation scans. The number of connected sections and the percentage of completion of the corridor was recorded in (Table 2). The mean and standard deviation $(\mathrm{M}+\mathrm{SD})$ and the range of connected values are shown.

Table 2. Ablation corridor assessment results. Patients with a corridor percentage higher than $70 \%$ are highlighted in bold. The bottom row shows the mean (M) and standard deviation (SD) of the percentage across all patients.

\begin{tabular}{lll}
\hline Patient & Percentage of corridor (\%) & Gap number \\
\hline 01 & 59.52 & 4 \\
02 & $\mathbf{9 2 . 6 4}$ & 2 \\
03 & $\mathbf{8 5 . 6 9}$ & 4 \\
04 & $\mathbf{7 3 . 4}$ & 2 \\
05 & $\mathbf{7 1 . 1 4}$ & 3 \\
06 & $\mathbf{7 1 . 0 5}$ & 3 \\
\hline & $\mathbf{7 5 . 6}+\mathbf{1 2}$ & {$[2,4]$} \\
\hline
\end{tabular}

Finally, a representative case is shown in Figures 4 and 5. Figure 4 presents the results for methods (i) scar area and (iii) comparison of ablation tissue between pre- and post-ablation. Figure 4 also presents the shell of the postablation scan with the signal intensity values of the preablation scan projected onto it, for a straightforward comparison. Figure 5 represents method (ii) comparing ablation lesions, where the signal intensity values of the shell have been deleted except on the exploration corridor.
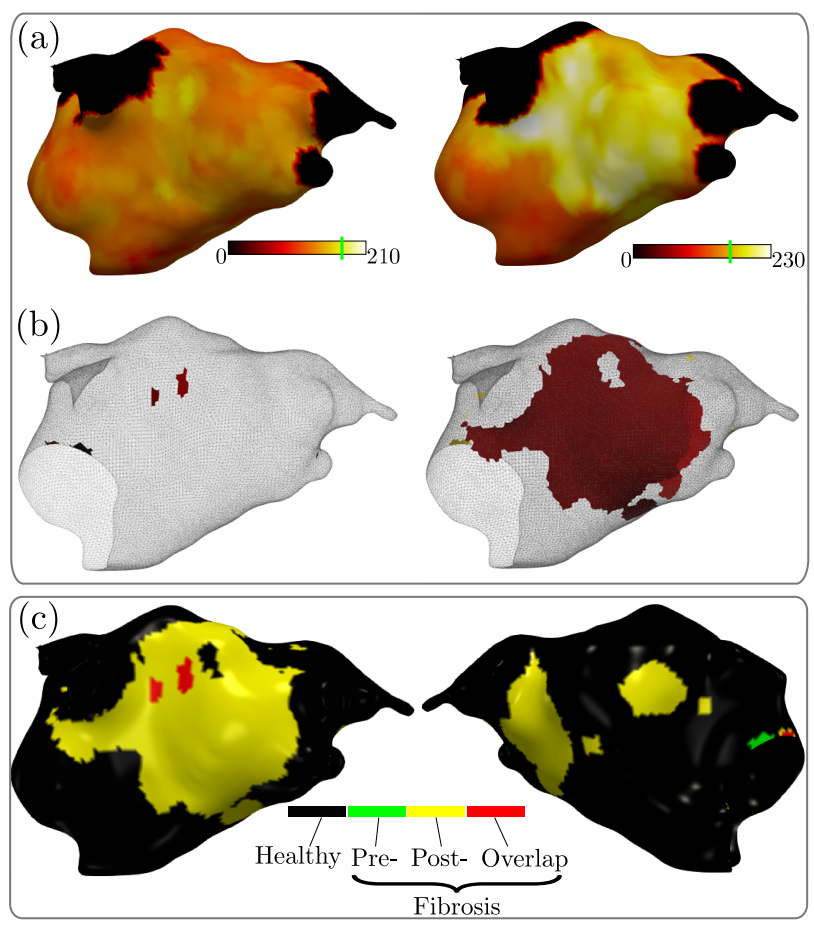

Figure 4. Results in a representative patient. (a) The pre- and post-ablation scans are shown. The thresholds are represented with a vertical green line on the colour bar. Notice the signal intensities from the pre- have been projected onto the postablation shell. (b) Shows the detected fibrotic tissue based on their respective thresholds. (c) Presents the scar tissue overlap between pre- and post-ablation scans as seen from the front and back. Black represents healthy tissue, green and yellow represent pre- and post-ablation fibrosis and red represents overlap.

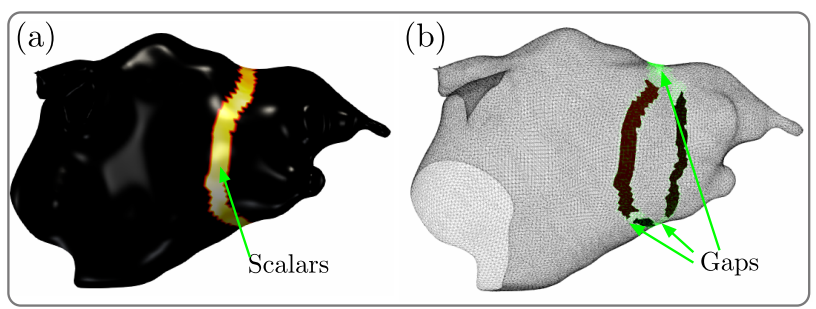

Figure 5. Visualisation of results in a representative patient. (a) Shows the ablation corridor, with the signal intensity values (scalars) are present only on the corridor. (b) Shows the fragments of the corridor where the signal intensity was higher than the selected threshold.

\section{Discussion}

The aim of this work was to provide a robust set of software tools to quantify ablation lesions, whilst comparing pre- and post-ablation scans. This paper has described the Advanced Scar Calculations pipeline, a set of semi-automatic tools to assess an ablation procedure outcome, which compares the states of the tissue before and after the procedure. The software tools were built on 
top of a stable and reliable platform.

Comparing scans, even of the same patient, is a difficult problem, it implies the comparison of two morphologically different shapes and a potentially complicated registration problem, such a limitation was reported on [1]. As seen in Figure 2, the shapes differ in the same patient, however not the physiological characteristics, a patient will have the same number of veins showing. In this work, the problem of matching two shapes from the same patient is overcome by projecting the signal intensity values from one shell onto another. In the tests made, this did not cause much ambiguity, except near the clipped veins, where the shapes would usually differ most. For example, the pre- shell in Figure 4(a) shows a relative resemblance to its counterpart in Figure 2 (top right).

While automating tasks is desirable in most cases, it can sometimes lead to difficult complications and a disassociation from the users. In this work, an effort was made to give the user necessary control. Firstly, the user would apply CemrgApp's capabilities to clip undesired elements of the physiology of the atrium; secondly, they would utilise the interactive tools in the Advanced Scar Calculations to assess the PVI success. For example, Figure 5 shows the case where the user could modify the threshold and assess whether the exploration corridor gets completed with a different threshold. Using the literature backed threshold values, the user could easily determine if the procedure was successful.

A limitation is the introduction of inter-user variability, for example, at the time of selecting the control points for the encirclement assessment, which could modify the ablation corridor percentage value.

\section{Conclusions}

The software presented is a semi-automated, user friendly framework where users are able to assess an ablation procedure outcome. The software is open source and available to download on version 2 of CemrgApp (https://github.com/CemrgAppDevelopers/CemrgApp/rel eases/tag/v2.0), as part of the Scar Quantification View. Future work will include a refinement of such tools to suggest encirclement control points to the user.

\section{References}

[1] M. Nuñez-Garcia, O. Camara, M. D. O’Neill, R. Razavi, H. Chubb, and C. Butakoff, "Mind the gap: Quantification of incomplete ablation patterns after pulmonary vein isolation using minimum path search," Medical Image Analysis, vol. 51, pp. 1-12, Jan. 2019, doi: 10.1016/j.media.2018.10.001.

[2] H. Chubb et al., "Optimization of late gadolinium enhancement cardiovascular magnetic resonance imaging of post-ablation atrial scar: a cross-over study," J Cardiovasc Magn Reson, vol. 20, no. 1, p. 30, May 2018, doi: 10.1186/s12968-018-0449-8.

[3] D. C. Peters et al., "Detection of pulmonary vein and left atrial scar after catheter ablation with threedimensional navigator-gated delayed enhancement MR imaging: Initial experience," Radiology, vol. 243, no. 3, pp. 690-695, Jun. 2007, doi: 10.1148/radiol.2433060417.

[4] Badger Troy J. et al., "Evaluation of left atrial lesions after initial and repeat atrial fibrillation ablation," Circulation: Arrhythmia and Electrophysiology, vol. 3, no. 3, pp. 249-259, Jun. 2010, doi: 10.1161/CIRCEP.109.868356.

[5] P. M. Halbfass, M. Mitlacher, O. Turschner, J. Brachmann, and C. Mahnkopf, "Lesion formation after pulmonary vein isolation using the advance cryoballoon and the standard cryoballoon: lessons learned from late gadolinium enhancement magnetic resonance imaging," Europace, vol. 17, no. 4, pp. 566-573, Apr. 2015, doi: 10.1093/europace/euu260.

[6] O. Razeghi et al., "CemrgApp: An interactive medical imaging application with image processing, computer vision, and machine learning toolkits for cardiovascular research," SoftwareX, vol. 12, p. 100570, Jul. 2020, doi: 10.1016/j.softx.2020.100570.

[7] I. Sim et al., "Reproducibility of atrial fibrosis assessment using CMR imaging and an open source platform," JACC: Cardiovascular Imaging, vol. 12, no. 10, pp. 2076-2077, Oct. 2019, doi: 10.1016/j.jcmg.2019.03.027.

[8] L. O'Neill et al., "Pulmonary vein encirclement using an Ablation Index-guided point-by-point workflow: cardiovascular magnetic resonance assessment of left atrial scar formation," Europace, vol. 21, no. 12, pp. 1817-1823, Dec. 2019, doi: 10.1093/europace/euz226.

[9] H. Chubb et al., "A comprehensive multi-index cardiac magnetic resonance-guided assessment of atrial fibrillation substrate prior to ablation: Prediction of long-term outcomes," Journal of Cardiovascular Electrophysiology, vol. 30 , no. 10, pp. 1894-1903, 2019, doi: $10.1111 /$ jce.14111.

[10] J. L. Harrison et al., "Cardiac magnetic resonance and electroanatomical mapping of acute and chronic atrial ablation injury: a histological validation study," Eur Heart J, vol. 35, no. 22, pp. 1486-1495, Jun. 2014, doi: 10.1093/eurheartj/eht560.

Address for correspondence:

José Alonso Solís-Lemus.

$4^{\text {th }}$ Floor North Wing, St. Thomas' Hospital, King's College London, Westminster Bridge Rd, Bishop's, London SE1 7EH jose.solislemus@kcl.ac.uk 